\title{
RYDBERG EXCITATION OF A BOSE-EINSTEIN CONDENSATE
}

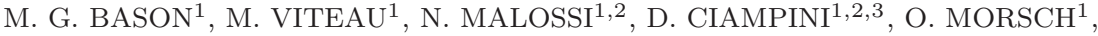 \\ E. ARIMONDO ${ }^{1,2,3}$ \\ ${ }^{1}$ CNR-INO, Dipartimento di Fisica E.Fermi, Università di Pisa, \\ Largo Pontecorvo 3, I-56127 Pisa, Italy \\ ${ }^{2}$ CNISM, Unitá di Pisa, Dipartimento di Fisica E.Fermi, \\ Largo Pontecorvo 3, I-56127 Pisa, Italy \\ ${ }^{3}$ Dipartimento di Fisica E.Fermi, Università di Pisa, \\ Largo Pontecorvo 3, I-56127 Pisa, Italy
}

\begin{abstract}
Rydberg excitation of an elongated Bose-Einstein condensate is performed. We show how the dipole-blockade mechanism restricts the number of Rydberg atoms it is possible to excite in such a cloud. Using the super-atom model we then look at the feasibility of observing coherent effects in even stricter geometries, one dimensional optical lattices.
\end{abstract}

\section{Introduction}

The prospect of exploiting Rydberg interactions to perform quantum-information processing has led to increased interest in Rydberg physics in recent years ${ }^{1,2,3}$. Alongside this field of interest, many other uses for Rydberg atoms have been proposed such as single-atom and single-photon sources ${ }^{2,4,5}$, generation of correlated photons $^{6}$, quantum simulation of complex spin systems ${ }^{7}$, creation of large optical nonlinearities ${ }^{8}$, creation of entangled many-particle states ${ }^{9}$ and measurement of electric fields ${ }^{10,11}$.

Many of these proposals rely upon the dipole blockade mechanism. This effect occurs when the excitation of an atom to a Rydberg state is inhibited if another, already excited, atom is less than the so-called blockade radius $r_{b}$ away ${ }^{12}$. As shown recently, the excitation of two individual atoms is a clear evidence for this effect ${ }^{13,14}$. For many more atoms the picture is very similar, we then have a collection of super-atoms ${ }^{15}$ in which a single excitation is shared among all the atoms inside the blockade radius ${ }^{16,17,18}$. Rydberg excitation blockade has previously been realised in disordered clouds of cold atoms ${ }^{19,20,21,22,23}$. One can add spatial order to the system by using an optical lattice. In such a system the trapped atoms are separated by a fixed distance, such that Rydberg excitation is possible at discrete frequencies ${ }^{24}$. 
In terms of quantum information, an array of trapped atoms coupled through longrange Rydberg interactions represents a scalable system ${ }^{3}$. To realise an array, one could use the Mott insulator phase of ultracold bosonic atoms, to load one atom in each site ${ }^{25}$. However Lukin et $\mathrm{al}^{26}$ suggest that experiments requiring single atoms excited to Rydberg states can also be performed with collective Rydberg excitations, each containing several hundreds or thousand of atoms. This makes it possible to work with Bose condensates loaded into optical lattices without the need to induce a Mott insulator transition in order to have single-atom occupation of the lattice sites.

This work reports the excitation of a Bose-Einstein condensate of rubidium atoms to Rydberg states. The dipole blockade phenomenon is investigated in one-dimensional geometries, both in absence and presence of an optical lattice.

\section{Apparatus}

The cold atom apparatus uses two quartz cells, which we will call "collection" and "science" respectively, horizontally mounted on a central metallic structure ${ }^{27}$. In the latter cell, Bose-Einstein condensates of around $10^{5}$ atoms of ${ }^{87} \mathrm{Rb}$ are created using a two-step evaporation protocol with a TOP-trap and a crossed optical dipole trap. These samples are then excited to Rydberg states with principal quantum number $n$ between 55 and 80 using a two-colour coherent excitation scheme. The first step uses light at $420 \mathrm{~nm}, 0.5-1 \mathrm{GHz}$ from the $5 S_{1 / 2} \rightarrow 6 P_{3 / 2}$ transition. The second step, infrared (IR) radiation laser at $1010-1030 \mathrm{~nm}$ is tuned to a specific $n$ state. To prevent long term drifts of the laser frequencies, the excitation lasers are stabilised to a scanning Fabry Perot interferometer, with a $780 \mathrm{~nm}$ laser locked to a $\mathrm{Rb}$ resonance acting as a reference.

By using an acousto-optic modulator, both the blue and IR laser light is typically pulsed for durations in the range of $0.5-10 \mu \mathrm{s}$. Following these pulses, the Rydberg atoms are ionised by applying a high voltage electric field to electrodes external to the quartz cell. After field ionisation, charge detection is performed using a channeltron. In order to avoid the formation of electric charges on the cell walls, which create uncontrolled electric fields, short pulses of a few $\mu$ s duration are used. Under such conditions a collection efficiency of $\eta=35 \pm 10$ was measured $^{28}$. This figure accounts for both the fraction of the produced charges transported to the channel electron multiplier through a two-stage acceleration and the multiplier detection efficiency.

The polarizations of both blue and IR lasers were linear and parallel to each other along the vertical axis. The excitation of the condensate to Rydberg states is performed $0.7 \mathrm{~ms}$ after the TOP magnetic field is switched off, with the atomic magnetic moments rotating in a plane containing the laser polarizazion axis. 


\subsection{D geometry}

Quasi one-dimensional atomic samples are created by ramping down one of the two trap beams of the crossed dipole trap in which the condensates are created. By letting the condensate expand for variable times (up to $500 \mathrm{~ms}$ ), one can vary the sample length $l$. The highly elongated clouds are up to $1 \mathrm{~mm}$ long, while their radial dimensions are on the order of $1-2 \mu \mathrm{m}$ (radial dipole trap frequencies are around $100 \mathrm{~Hz}$ ). To avoid excitation of the cloud the power in the beam is ramped down slowly (around $40 \mathrm{~ms}$ ), while the other is increased to keep the radial trap frequency constant. In these experiments it was important that the radial size of the condensate was smaller than the expected blockade radii. This is indeed possible as the expected blockade radii are in the range of $5-15 \mu \mathrm{m}$.

The Rabi frequencies for the blue and IR lasers are $\Omega_{\text {blue }} / 2 \pi$ up to $80 \mathrm{MHz}$ and $\Omega_{\mathrm{IR} / 2 \pi}$ around $10 \mathrm{MHz}$, respectively. For a blue laser detuning of $1 \mathrm{GHz}$, the twophoton Rabi frequency is in the $20-100 \mathrm{kHz}$ range. Both blue and IR excitation beams were aligned so as to be almost parallel to the dipole trap beam in which the condensate expands. They are focused to a waist larger than the atomic cloud size, the minimum being $100 \mu \mathrm{m}$. Thus the ultracold cloud is within the beam waist even in the presence of fluctuations in the spatial position of the cold atomic cloud.

Figure 1 shows the results of an excitation experiment for the $66 D_{5 / 2}$ state using a $1 \mu \mathrm{s}$ excitation pulse, in which a linear increase of the number of Rydberg atoms with the length of the condensate is visible. This result agrees with the simple intuitive picture of super-atoms, with dimension twice the $r_{b}$ blockade radius, being closely packed in a one-dimensional array of varying length.

Extracting the mean distance between Rydberg atoms from the data in figure 1 is thus a measure of the blockade radius. In doing so, one considers that in the saturated regime the individual superatoms perform Rabi oscillations between their ground and excited states, thus on average only half of the excitable super-atoms are detected. For the data in figure 1, this gives $7.2 \pm 1.7 \mu \mathrm{m}$; changing the Rydberg

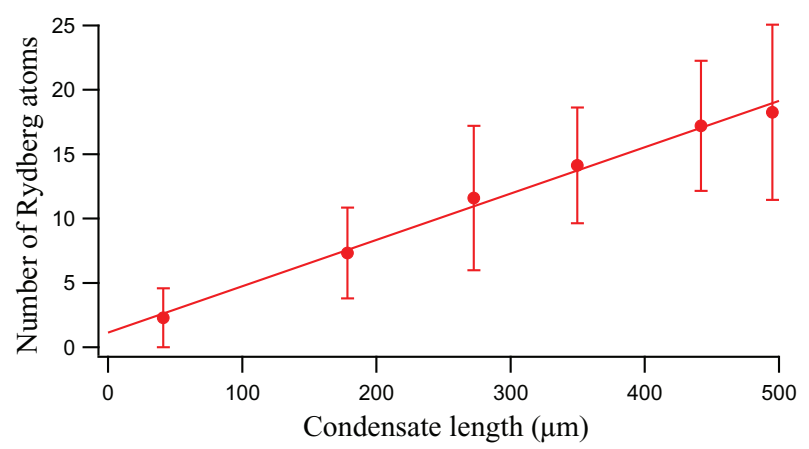

Fig. 1. Increasing number of $66 \mathrm{D}_{5 / 2}$ Rydberg atoms as a function of condensate size. The error bars represent the standard deviation of the data. 
state allows one to measure the scaling of blockade radius with principal quantum number ${ }^{30}$.

\section{2. $1 D$ optical lattice}

In an optical lattice, neutral atoms are trapped in the intensity maxima or minima of a standing wave light field owing to the optical dipole force. For a red-detuned light field, the maxima give rise to lattice sites which, in the case of a counterpropagating lattice, are separated by $\lambda / 2$, where $\lambda$ is the wavelength of the optical lattice light. One would like to be able to selectively address individual sites. Most laser sources, with wavelengths in the range of $0.5-1.5 \mu \mathrm{m}$, impose lattice spacings of around $1 \mu \mathrm{m}$ making the addressing of single sites technically difficult. Another approach is to use long wavelength lasers, as $\mathrm{CO}_{2}$ lasers, which operate at $10.6 \mu \mathrm{m}$. The lifetime of Rydberg states in such lattices however, are prohibitively short for quantum information purposes ${ }^{29}$.

Alternatively, optical lattices with a large lattice spacing can be formed using an angle-tuned geometry. In such cases, the periodic potential created by two linearly polarised laser beams propagating at an angle $\phi$ result in a lattice constant is $d_{L}=$ $\lambda /(2 \sin (\phi / 2))$. In our experiment this allows the lattice spacing to be tuned from $420 \mathrm{~nm}$ to around $25 \mu \mathrm{m}$, see figure 2 . This lattice is added along the direction of the expanded BEC.

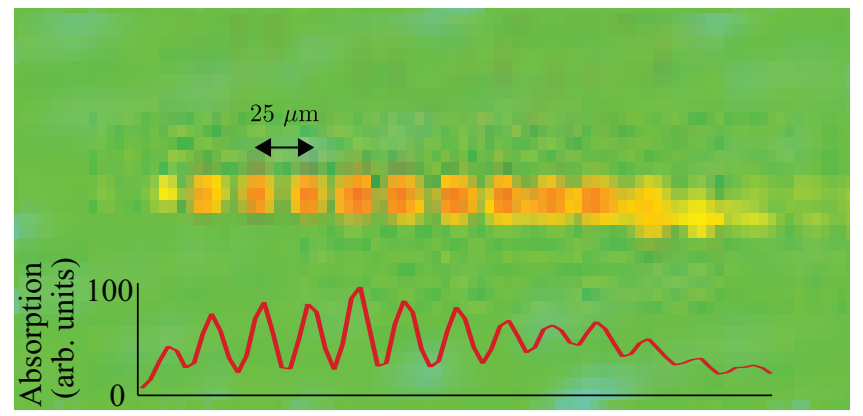

Fig. 2. An in-situ absorption image of the atoms trapped in the optical lattice. Knowing the size of each pixel enables the calibration of the lattice constant $d_{L}$. Note that visibility towards the edge of the lattice is reduced due to the finite size of the lattice beams and the smaller atom number.

For the largest spacings, the depth $V_{0}$ of the periodic potential $V(x)=$ $V_{0} \sin \left(2 \pi x / d_{L}\right)$ measured in units of the recoil energy $E_{\text {rec }}=\hbar^{2} \pi^{2} /\left(2 m d_{L}^{2}\right)$ (where $m$ is the mass of the atoms) is around $V_{0} / E_{\mathrm{rec}} \sim 15000$. In the lattice direction the size of the on-site wavefunction is around $\Delta x=d_{L} /\left(\pi\left(V_{0} / E_{\mathrm{rec}}\right)^{1 / 4}\right) \sim 0.05-0.4 \mu \mathrm{m}$. Thus the individual mini-condensates created by the lattice are both well separated and their longitudinal size of each condensate is much less than $r_{b}$. It should be possible to then focus the Rydberg excitation beams so that as few as one 
mini-condensate performs Rabi oscillations. One would have an array of quasi zerodimensional systems as the spatial extension of the ground-state wavefunction is smaller than $r_{b}$ in all directions.

Importantly, the effect of Rydberg excitation on the condensate phase coherence of atoms in optical lattices needs to be considered. If one would like to perform multiple experimental cycles on the same sample, then as little modification to the sample as possible is desired. Previously we have shown that more than 10 excitation-detection cycles can be performed on the same condensate without a noticeable loss of phase coherence or atom number in the condensate ${ }^{30}$.

\subsection{Simulations}

As a stepping stone towards more demanding applications such as quantum computing, it is desirable to observe clear signs of the coherent nature of Rydberg excitation. Due to the different number of atoms in each lattice site of the optical lattice each super-atom should Rabi flop at a different rate. The effect of averaging over the dynamics of each super-atom is that the Rabi oscillations wash out. A simple numerical model provides some indication of the range of parameters necessary to observe coherent oscillations. We consider the number of ground-state atoms in each lattice site, $N_{i}$, according to a Gaussian distribution. Each lattice sites also experiences a different Rabi-frequency due to the intensity distribution of the excitation lasers which we also assume to be Gaussian. Averaging over these two distributions, we sum the probability of there being a Rydberg atom in a single site and thus arrive at the total number of Rydberg atoms expected.

Flat-top beams have previously been used to excite Rydberg atoms ${ }^{31}$, but are technically challenging to achieve on the scale of $\sim 10 \mu \mathrm{m}$. According to the superatom picture, the collective Rabi frequency for a single lattice site, $i$, containing $N_{i}$ atoms excitation is,

$$
\Omega_{\text {coll }}=\sqrt{N_{i}} \Omega_{\text {single }},
$$

Typically the single-particle Rabi frequency for our experimental parameters is $\Omega_{\text {single }} \sim 2 \pi \times 200 \mathrm{kHz}$. A small dephasing rate between lattice sites, is added chosen so as to reproduce the observed long-term increase in the number of collective excitations ${ }^{30}$. Also built into the model is the relative position between the excitation beam and the optical lattice. Figure 3 shows the number of Rydberg atoms excited as a function of time for various beam sizes. Critically a reduction from 20 to $15 \mu \mathrm{m}$ has a marked effect on the visibility of the oscillation. This suggests that the requirement of being slightly smaller than the lattice constant is not sufficient, and to clearly observe coherent oscillations a tighter focussed beam is critical. Moving towards single site addressability with focussed Rydberg excitation beams implies that the absolute positioning of the lattice relative to the beams becomes important. The effect of a jitter in the lattice position is shown in figure 4. Many 
experimental cycles are needed to detect an oscillation, thus it is important that the position stability of the lattice be present in any simulation. This effect is modelled by introducing a random position fluctuation of variable size.

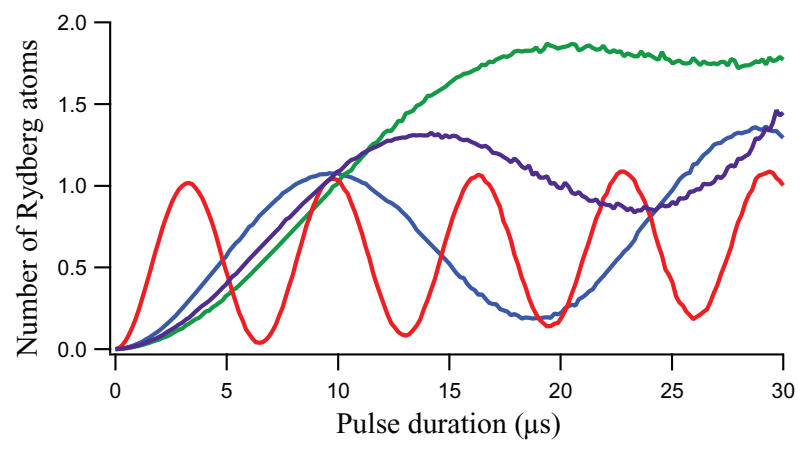

Fig. 3. Simulated Rabi oscillations in an optical lattice for various excitation beam sizes: Gaussian waists of 5, 15, 20 and $25 \mu \mathrm{m}$ for the red, blue, purple and green lines, respectively. Lattice constant $25 \mu \mathrm{m}, 2000$ atoms in total, blockade radius of $10 \mu \mathrm{m}$ and radial width of $2 \mu \mathrm{m}$. Jitter in the lattice position is modelled as a uniform random distribution with a width of $1 \mu \mathrm{m}, 250 \mu \mathrm{m}$ longitudinal width $(1 / \mathrm{e})$ of the condensate.

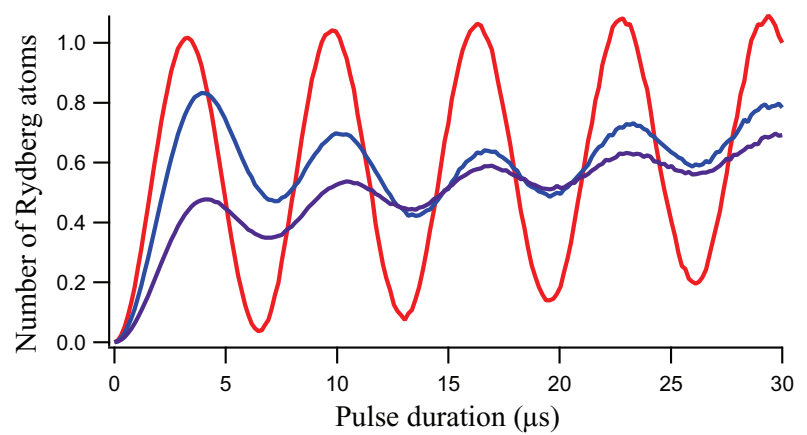

Fig. 4. The effect of a relative offset in the lattice position with respect to the excitation beam, jitter in the lattice position of 1,5 and $10 \mu \mathrm{m}$ for the red, blue and purple lines respectively. Lattice constant $25 \mu \mathrm{m}$, beam waist, $5 \mu \mathrm{m}$. 2000 atoms in total, blockade radius of $10 \mu \mathrm{m}$, radial width of $2 \mu \mathrm{m}$ and longitudinal condensate width of $250 \mu \mathrm{m}$.

\section{Conclusions}

Through modelling the excitation dynamics of Rydberg atoms trapped in an largeperiod optical lattice, we have clarified the importance of various experimental parameters. Critically, any fluctuation in the lattice position manifests itself as a reduction in the visibility of the coherent dynamics. While various technical challenges 
are still to be met, this work provides an insight into the feasability of detecting Rabi oscillations in one-dimensional optical lattices. Progress in experiments should open up interesting avenues such as the production of highly entangled states ${ }^{46}$, with applications in scalable quantum information with Rydberg atoms ${ }^{3}$.

\section{Acknowledgments}

Financial support by the EU-STREP "NAMEQUAM" and by a CNISM "Progetto Innesco 2007" grant is gratefully acknowledged.

\section{References}

1. D. Jaksch, J. I. Cirac, P. Zoller, S. L. Rolston, R. Côté and M. D. Lukin, Phys. Rev. Lett. 85, 2208 (2000).

2. M. D. Lukin, M. Fleischhauer, and R. Côté, L. M. Duan, D. Jaksch, J. I. Cirac, and P. Zoller, Phys. Rev. Lett. 87, 037901 (2001).

3. M. Saffman, T. G. Walker, and K. Mølmer, Rev. Mod. Phys. 82, 2313 (2010).

4. M. Saffman, and T. G. Walker, Phys. Rev. A 66, 065403 (2002).

5. T. Pohl, E. Demler, and M.D. Lukin, Phys. Rev. Lett. 104, 043002 (2010).

6. J. D. Pritchard, C. S. Adams and K. Mølmer, arXiv:1108.5165 (2011).

7. H. Weimer, M. Müller, I. Lesanovsky, P. Zoller, and H. P. Büchler, Nature Phys. 6, $382(2010)$.

8. A. K. Mohapatra, M. G. Bason, B. Butscher, K. J. Weatherill, and C. S. Adams, Nature Phys. 4, 890 (2008).

9. B. Olmos, R. González-Férez, and I. Lesanovsky, Phys. Rev. Lett. 103, 185302 (2009).

10. A. Tauschinsky, R M. T. Thijssen, S. Whitlock, H. B. van Linden van den Heuvell, and R. J. C. Spreeuw, Phys. Rev. A 81, 0634112010.

11. R. P. Abel, C. Carr, U. Krohn, and C. S. Adams, Phys. Rev. A 84, 023408 (2011).

12. D. Comparat and P. Pillet, J. Opt. Soc. Am. B 27, A208, (2010).

13. E. Urban, T. A. Johnson, T. Henage, L. Isenhower, D. D. Yavuz, T. G.Walker and M. Saffman, Nature Physics, 5, 110 (2009).

14. A. Gaëtan, Y. Miroshnychenko, T. Wilk, A. Chotia, M. Viteau, D. Comparat P. Pillet, A. Browaeys, and P. Grangier, Nature Physics, 5, 115 (2009).

15. V. Vuletic, Nature Phys. 2, 801 (2006).

16. F. Robicheaux and J. Hernández, Phys. Rev. A 72, 063403 (2005).

17. R. Heidemann, U. Raitzsch, V. Bendkowsky, B. Butscher, R. Löw, L. Santos, and T. Pfau, Phys. Rev. Lett. 99, 163601 (2007).

18. R. Stanojevic, and R. Côté, Phys. Rev. A 80, 033418 (2009).

19. D. Tong, S.M. Farooqi, J. Stanojevic, S. Krishnan, Y. P. Zhang, R. Côté, E. E. Eyler, and P. L. Gould, Phys. Rev. Lett. 93, 063001 (2004).

20. K. Singer, M. Reetz-Lamour, T. Amthor, L. G. Marcassa, and M. Weidemüller, Phys. Rev. Lett. 93, 163001 (2004).

21. K. Afrousheh, P. Bohlouli-Zanjani, D. Vagale, A. Mugford, M. Fedorov, and J. D. D. Martin. Phys. Rev. Lett. 93, 233001 (2004).

22. T. Cubel Liebisch, A. Reinhard, P. R. Berman, and G. Raithel, Phys. Rev. Lett. 95, 253002 (2005) and ibidem 98, 109903 (2007).

23. T. Vogt, M. Viteau, J. Zhao, A. Chotia, D. Comparat, and P. Pillet, Phys. Rev. Lett. 97, 083003 (2006).

24. M. Mayle, W. Zeller, N. Tezak, and P. Schmelcher, Phys. Rev. A 84, 010701 (2011). 
25. M. Greiner, O. Mandel, T. Esslinger, T. W. Hänsch and I. Bloch, Nature 415, 39 (2002)

26. M.D. Lukin, et al., Phys. Rev. Lett. 87, 037901 (2001).

27. H. Lignier, C. Sias, D. Ciampini, Y. Singh, A. Zenesini, O. Morsch, and E. Arimondo, Phys. Rev. Lett. 99, 220403 (2007).

28. M. Viteau, J. Radogostowicz, A. Chotia, M. Bason, N. Malossi, F. Fuso, D. Ciampini, O. Morsch, I. I. Ryabtsev, and E.Arimondo, J. Phys. B: At. Mol. Opt. Phys. 43, 155301 (2010).

29. R. M. Potvliege and C. S. Adams, New. J. Phys. 8, 163 (2006).

30. M. Viteau, M. G. Bason, J. Radogostowicz, N. Malossi, D. Ciampini, O. Morsch, and E. Arimondo, Phys. Rev. Lett. 107, 060402 (2011)

31. M. Reetz-Lamour, T. Amthor, J. Deiglmayr, and M. Weidemller, Phys. Rev. Lett. 100, 253001 (2008).

32. T. Wilk, A. Gaëtan, C. Evellin, J. Wolters, Y. Miroshnychenko, P. Grangier, and A. Browaeys, Phys. Rev. Lett. 104, 010502 (2010).

33. L. Isenhower, E. Urban, X.L. Zhang, A.T Gill, T. Henage, T. A. Johnson, T. G. Walker, and M. Saffman, Phys. Rev. Lett. 104, 010503, (2010).

34. R. Heidemann, U. Raitzsch, V. Bendkowsky, B. Butscher, R. Löw, and T. Pfau, Phys. Rev. Lett. 100, 033601 (2008).

35. C. S. E. van Ditzhuijzen, A. F. Koenderink, J.V. Hernańdez, F. Robicheaux, L. D. Noordam, and H. B. van Linden van den Heuvell, Phys. Rev. Lett. 100, 243201 (2008).

36. E. Courtade, M. Anderlini, D. Ciampini, J. H. Müller, O. Morsch, E. Arimondo, M. Aymar and E. J. Robinson, J. Phys. B: At. Mol. Opt. Phys. 37, 967 (2004).

37. M. Saffman and T. G. Walker, Phys. Rev. A 72, 022347 (2005).

38. Wenhui Li, P.L. Tanner, and T. F. Gallagher, Phys. Rev. Lett. 94, 173001 (2005).

39. Beterov I I, Ryabtsev I I, Tretyakov D B and Entin V M Phys. Rev. A 79052504 (2009).

40. B. K. Teo, D. Feldbaum, T. Cubel, J. R. Guest, P. R. Berman, and G. Raithel, Phys. Rev. A 68, 053407 (2003).

41. Wenhui Li, I. Mourachko, M.W. Noel, and T. F. Gallagher, Phys. Rev. A 67, 052502 (2003).

42. T. Walker and M. Saffman, Phys. Rev. A 77, 032723 (2008).

43. C. Ates, T Pohl, T Pattard and J M Rost, J. Phys. B: At. Mol. Phys. 39, L233 (2006).

44. R. Löw, H. Weimer, U. Krohn, R. Heidemann, V. Bendkowsky, B. Butscher, H. P. Büchler, and T. Pfau, Phys. Rev. A 80, 033422 (2009).

45. I. Lesanovsky, Phys. Rev. Lett. 106, 025301 (2011), and references therein.

46. Z. Zuo and K. Nakagawa, Phys. Rev. A 82, 062328 (2010). 